\title{
Glucose Metabolism in the Insula and Cingulate Is Affected by Systemic Inflammation in Humans
}

\author{
Jonas Hannestad ${ }^{1}$, Kalyani Subramanyam ${ }^{1}$, Nicole DellaGioia ${ }^{1}$, Beata Planeta-Wilson ${ }^{2}$, David Weinzimmer ${ }^{2}$, \\ Brian Pittman ${ }^{1}$, and Richard E. Carson ${ }^{2}$ \\ ${ }^{1}$ Department of Psychiatry, Yale School of Medicine, New Haven, Connecticut; and ${ }^{2}$ Department of Diagnostic Radiology, Yale \\ School of Medicine, New Haven, Connecticut
}

\begin{abstract}
Depression is associated with systemic inflammation, and the systemic inflammation caused by endotoxin administration elicits mild depressive symptoms such as fatigue and reduced interest. The neural correlates of depressive symptoms that result from systemic inflammation are poorly defined. The aim of this study was to use ${ }^{18} \mathrm{~F}-\mathrm{FDG}$ PET to identify brain regions involved in the response to endotoxin administration in humans. Methods: Nine healthy subjects received double-blind endotoxin $(0.8 \mathrm{ng} / \mathrm{kg})$ and placebo on different days. ${ }^{18} \mathrm{~F}-\mathrm{FDG}$ PET was used to measure differences in the cerebral metabolic rate of glucose in the following regions of interest: insula, cingulate, and amygdala. Serum levels of tumor necrosis factor- $\alpha$ and interleukin- 6 were used to gauge the systemic inflammatory response, and depressive symptoms were measured with the Montgomery-Åsberg Depression Rating Scale and other scales. Results: Endotoxin administration was associated with an increase in Montgomery-Åsberg Depression Rating Scale, increased fatigue, reduced social interest, increased levels of inflammatory cytokines, higher normalized glucose metabolism (NGM) in the insula, and, at a trend level, lower NGM in the cingulate. Secondary analyses of insula and cingulate subregions indicated that these changes were driven by the right anterior insula and the right anterior cingulate. There was a negative correlation between peak cytokine levels and change in social interest and between peak cytokine levels and change in insula NGM. There was a positive correlation between the change in NGM in the insula and change in social interest. Conclusion: Systemic inflammation in humans causes an increase in depressive symptoms and concurrent changes in glucose metabolism in the insula and cingulate-brain regions that are involved in interoception, positive emotionality, and motivation.
\end{abstract}

Key Words: ${ }^{18}$ F-FDG PET; endotoxin; inflammation; depression; insula

J Nucl Med 2012; 53:601-607

DOI: 10.2967/jnumed.111.097014

\footnotetext{
Received Aug. 15, 2011; revision accepted Nov. 29, 2011.

For correspondence or reprints contact: Jonas Hannestad, Psychiatry

Ribicoff Research, New Haven, CT 06520-8068.

E-mail: jonas.hannestad@yale.edu

Published online Mar. 13, 2012.

COPYRIGHT (c) 2012 by the Society of Nuclear Medicine, Inc.
}

D epression is a common and devastating illness caused by interactions between stress and genetic and psychologic susceptibility factors $(1,2)$. Depression is associated with impaired neuroplasticity and production of inflammatory cytokines in the brain $(1,3)$. Depression is also associated with increased systemic levels of inflammatory cytokines, including tumor necrosis factor- $\alpha(\mathrm{TNF} \alpha)$ and interleukin-6 (IL-6) (4). It is possible that systemic inflammation contributes to depressive symptoms, especially the neurovegetative symptoms of depression $(5,6)$. This notion is supported by the fact that inflammatory cytokines cause depressive-like behaviors in rodents (7) and that, in humans, inflammatory cytokines are associated with depressive symptoms during infections (8), during treatment with interferon- $\alpha$ (5), and after administration of acute inflammatory stimuli $(9-11)$.

The neural circuits involved in the brain's response to systemic inflammation have not been well characterized. Imaging using ${ }^{18}$ F-FDG PET or functional MRI has implicated regions such as the insula and anterior cingulate in experimental asthma exacerbation (12), during a social rejection paradigm in which subjects received endotoxin (10), in typhoid-vaccine-induced fatigue (13), and depressed mood during an emotional face-perception task (14). Other regions include the nucleus accumbens and putamen in interferon- $\alpha$-induced fatigue (15), the substantia nigra in typhoid-vaccine-induced cognitive impairment (16), and the ventral striatum in response to monetary reward cues when subjects received endotoxin (17). Most of these studies measured depressive symptoms that occurred as an interaction between inflammation and a behavioral paradigm without identifying neural correlates of depressive symptoms that occur as a result of systemic inflammation per se. We found that endotoxin administration causes mild depressive symptoms, particularly fatigue and reduced social interest (11). The aim of this study was to use ${ }^{18} \mathrm{~F}-\mathrm{FDG}$ PET to examine the neural correlates of these endotoxin-induced symptoms. On the basis of these studies and studies of idiopathic depression, we hypothesized that endotoxin administration would be associated with changes in glucose metabolism in the insula, cingulate, and amygdala. 


\section{MATERIALS AND METHODS}

\section{Study Design}

In this double-blind, randomized, placebo-controlled, crossover study, 9 healthy subjects received an intravenous dose of endotoxin $(0.8 \mathrm{ng} / \mathrm{kg}$ of body weight) on one day and placebo (saline) on another day, separated by $1 \mathrm{wk}$. Brain glucose metabolism was measured with ${ }^{18}$ F-FDG PET, with tracer injection 90 min after endotoxin or saline administration, when the systemic immune response peaked (18). The study was approved by the Yale University Human Research Protection Program, and informed consent was obtained from all subjects.

\section{Subjects}

Healthy subjects ( 1 woman, 8 men; mean age \pm SD, $28.6 \pm 8.2$ y) underwent screening with the structured clinical interview for the Diagnostic and Statistical Manual of Mental Disorders, 4th edition (19), to exclude those with psychiatric conditions. Urine drug and alcohol screens were used to exclude substance misuse. Medical screening included physical and neurologic examination; complete blood count; chemistries ( $\mathrm{Na}, \mathrm{K}, \mathrm{Cl}, \mathrm{Ca}$, phosphate, glucose, blood urea nitrogen, and creatinine); liver and thyroid function tests; urinalysis; C-reactive protein; serologies for HIV, syphilis, and viral hepatitis; and electrocardiogram. Subjects could not participate if they had had an infection or vaccination in the last month or if they had partaken of substance misuse in the last 6 mo. Subjects were asked not to take any over-the-counter nonsteroidal antiinflammatory drugs or drink alcohol for $48 \mathrm{~h}$ before each scan day. Subjects were recruited by advertisement from the greater New Haven area.

\section{Endotoxin Administration}

On each study day, subjects came to the PET Center in the morning after an overnight fast. After a urine drug screen and pregnancy test, vital signs were recorded. Two intravenous catheters were used, 1 for hydration and administration of endotoxin and ${ }^{18} \mathrm{~F}-\mathrm{FDG}$ and 1 for blood sampling. Clinical Center Reference Endotoxin for human use was provided by the National Institutes of Health and administered under a Food and Drug Administration Investigational New Drug application. Cardiac rhythm was continuously monitored. Heart rate and blood pressure were recorded every $15 \mathrm{~min}$ for the first $2 \mathrm{~h}$, then every $30 \mathrm{~min}$ thereafter. Mean arterial pressure was estimated as follows: mean arterial pressure $=2 / 3 \times$ diastolic blood pressure $+1 / 3 \times$ systolic blood pressure. Body temperature was recorded hourly.

\section{MRI}

To allow coregistration of PET and MR images for analysis, each subject underwent an MRI scan on a 3-T whole-body scanner (Trio; Siemens Medical Systems) with a circularly polarized head coil. The following magnetization-prepared rapid-acquisition with gradient echo sequence was used: sagittal 3-dimensional turbo flash; 250-mm field of view; 1-mm-thick slices; 176 slices total; echo time, 2.78; repetition time, 2,500; inversion time, 1,100; flip angle, 7 ; matrix, $256 \times 256$; and number of signal averages, 2 . The dimension and pixel size of MR images were $256 \times 256 \times 176$ and $0.98 \times 0.98 \times 1.0 \mathrm{~mm}$, respectively.

\section{PET}

PET data were collected using an ECAT EXACT HR + PET scanner (Siemens). Left ventricular (LV) input function was obtained from dynamic PET of the heart in 2-dimensional mode for $35 \mathrm{~min}$ (ten 30-s and ten 3-min frames). After reconstruction,
4 LV slices were identified for region-of-interest (ROI) placement. The 0- to 5-min frames were averaged to locate the LV blood pool, and the 25- to 35-min frames allowed identification of regions of myocardial ${ }^{18}$ F-FDG uptake. A circular ROI was placed over the LV chamber on each of the 4 images (LV image blood pool myocardial ${ }^{18} \mathrm{~F}$-FDG uptake) such that spillover from the myocardium was minimized. The LV time-activity curve was obtained from the average of time-activity curves derived from the ROI in each of the 4 slices. Venous blood samples were acquired beginning at $15 \mathrm{~min}$ after ${ }^{18} \mathrm{~F}-\mathrm{FDG}$ injection. The venous sample at 50 min was scaled by the ratio of the average LV radioactivity concentration to that in venous samples from 25 to $35 \mathrm{~min}$ after ${ }^{18} \mathrm{~F}-\mathrm{FDG}$ injection, to extend the input function to include the period of brain imaging. After cardiac imaging, a 10-min emission and an 8-min transmission brain scan were acquired, beginning 45 min after ${ }^{18}$ F-FDG injection. The complete input function and plasma glucose concentration were then used to generate parametric images of glucose metabolism rate (20). Global cerebral metabolic rate of glucose (CMRGlu) and regional CMRGlu (mg/ $100 \mathrm{~g} / \mathrm{min}$ ) were measured from these images.

\section{ROI Analysis}

MRI-based ROI analysis was used for the following a priori ROIs: insula, cingulate, and amygdala, which were defined using an automated anatomic labeling template (21) in Montreal Neurologic Institute space (22). The insula was divided into anterior and posterior regions along the central sulcus of the insula (23). For each subject, the PET image was coregistered to the T1weighted 3-T MR image using a 6-parameter mutual information algorithm (24) (FSL-FLIRT, version 3.2; Analysis Group, FMRIB), which was subsequently coregistered to the automated anatomic labeling template in Montreal Neurologic Institute space using a nonlinear transform with Bioimage Suite (version 2.5; Yale University). Mean ROI values were generated in regional CMRGlu images using the combined transformation from template to PET space. Normalized regional CMRGlu was calculated by dividing the absolute regional values by the global CMRGlu value. Differences in normalized glucose metabolism (NGM) between the placebo and endotoxin condition were tested. In addition to ROI analysis, statistical parametric mapping (SPM), described in the supplemental data (supplemental materials are available online only at http://jnm.snmjournals.org), was used to identify additional regions affected by systemic inflammation.

\section{Behavioral Assessments}

The primary behavioral outcome was the Montgomery-Åsberg Depression Rating Scale (MADRS). Secondary outcomes included the MADRS lassitude item, which measures motivation and fatigue; the fatigue subscale of the Profile of Mood States (25); a visual-analog scale to measure self-reported social interest (11); and scales to rate headache, muscle aches, chills, and nausea on a scale from 0 to 4 . The MADRS was assessed at baseline and at $120 \mathrm{~min}$ after endotoxin or saline administration; all other behavioral measures were assessed at baseline and at 60, 90, 120, and $180 \mathrm{~min}$. Subjects were asked how they felt in the previous hour.

\section{Immunologic and Endocrine Measures}

Blood samples for immune and endocrine measures were obtained at baseline and 60, 90, 120, and $180 \mathrm{~min}$ after endotoxin or placebo administration. Quantitative sandwich enzyme immunoassays (Quantikine; R\&D Systems, Inc.) were used to measure 
levels of TNF $\alpha$ and IL-6. The coefficients of variation were $1.9 \%$ and $1.7 \%$, respectively. Plasma cortisol was measured with a solid-phase radioimmunoassay (Siemens).

\section{Statistical Analysis}

Outcomes were summarized descriptively and assessed for normality before analysis using normal probability plots and Kolmogorov test statistics. The change in each dependent variable (behavioral measures and cytokines) was assessed using linear mixed models, which included condition (placebo, endotoxin) and time $(0,60,90,120$, and $180 \mathrm{~min} ; 0$ and $120 \mathrm{~min}$ for MADRS) as within-subject factors and subject as a clustering factor. ROI analyses included condition and region as within-subject factors, and regions were modeled as within-subject factors using random subject effects and structured variance-covariance patterns. Nonnormally distributed outcomes were analyzed with the same factors, using a nonparametric approach for repeated-measures data (26), in which the data were first ranked and then fitted using a mixed-effects model with an unstructured variance-covariance matrix and $P$ values adjusted for ANOVA-type statistics (ATS). Association between NGM, cytokines, and behavioral measures were assessed using Spearman correlations. All tests are 2-sided and considered statistically significant at an $\alpha$-value of 0.05 after correction for multiple comparisons. The primary analysis was corrected for multiple tests for the 3 a priori ROIs. Secondary ROI analyses of insula and cingulate subregions were adjusted for multiple tests within each region (4 and 6, respectively). All analyses were performed using SAS software (version 9.1; SAS Institute Inc.).

\section{RESULTS}

\section{Physiologic Effects}

There was a condition-by-time interaction on mean arterial pressure $\left(F_{4,40}=25, P<0.0001\right)$, because it increased with endotoxin (from $95 \pm 6$ to $100 \pm 7 \mathrm{~mm} \mathrm{Hg}$ ) but not with the placebo. There was a significant increase in heart rate over time $\left(F_{4,36}=8, P=0.0001\right)$ in both conditions. Although the increase in heart rate in the endotoxin condition ( $62 \pm 15$ to $75 \pm 19 \mathrm{bpm} ; 20 \%$ ) was more pronounced than in the placebo condition $(56 \pm 18$ to $62 \pm 15$;
$11 \%$ ), the main effect and the condition-by-time interactions were not significant. Similarly, although endotoxin caused an increase in body temperature $\left(96.9^{\circ} \mathrm{F} \pm 1.3^{\circ} \mathrm{F}\right.$ to $98.8^{\circ} \mathrm{F} \pm 1.2^{\circ} \mathrm{F}$ ), compared with the placebo condition $\left(97.2^{\circ} \mathrm{F} \pm 0.8^{\circ} \mathrm{F}\right.$ to $\left.97.6^{\circ} \mathrm{F} \pm 0.8^{\circ} \mathrm{F}\right)$, there was no significant main effect or condition-by-time effect on temperature $\left(F_{1,1}=2.8, P=0.3\right.$, and $\left.F_{4,4}=1.1, P=0.5\right)$.

\section{Immunologic and Endocrine Effects}

There was a condition-by-time interaction on TNF $\alpha$ levels $\left(F_{4,70}=5.3, P<0.001\right)$, because TNF $\alpha$ levels increased at each time after baseline (60, 90, 120, and $180 \mathrm{~min}$; all $P<0.002)$. In addition, there was a condition-by-time interaction on IL-6 levels $\left(F_{4,70}=27.5, P<\right.$ $0.001)$, because IL-6 levels increased at each time after baseline $(60,90,120$, and $180 \mathrm{~min}$; $P$ range $0.03-.0001)$. TNF $\alpha$ and IL-6 levels were highly correlated $(r=0.95$, $P<0.0001)$. Cortisol levels did not change over time with placebo administration, whereas they increased 2-fold to a peak of $33.4 \pm 5.9 \mathrm{mg} / \mathrm{mL}$ at $180 \mathrm{~min}$ (versus placebo, $p=0.00001$ ) after endotoxin administration. There were no order effects on immune or endocrine measures.

\section{Behavioral Effects}

The MADRS (Fig. 1A) increased from $0.4 \pm 0.2$ to $4.9 \pm$ 1.8 after endotoxin administration, whereas no change $(0.6 \pm 0.3$ to $0.9 \pm 0.5)$ was observed with placebo (interaction, $\left.F_{1,24}=7.1, P=0.013\right)$. There was a conditionby-time interaction on MADRS lassitude score $\left(F_{1,24}=6.0\right.$, $P=0.022)$ because it increased with endotoxin $(0.2 \pm 0.4$ to $1.9 \pm 1.8)$ but not with placebo $(0.3 \pm 0.5$ to $0.6 \pm 0.9)$. There was a condition-by-time interaction on the Profile of Mood States fatigue score (Fig. 1B; nonparametric: ATS, 3.0; numerator degrees of freedom, 2.5; $P=0.04$ ), with significant increases due to endotoxin at $180 \mathrm{~min}(1.9 \pm 3.9$ to $5.2 \pm 4.6$; ATS, 6.3; numerator degrees of freedom, 1 ; $P=0.012)$ but not with placebo $(2.9 \pm 4.0$ to $1.4 \pm 1.6)$. There was a significant condition-by-time interaction on

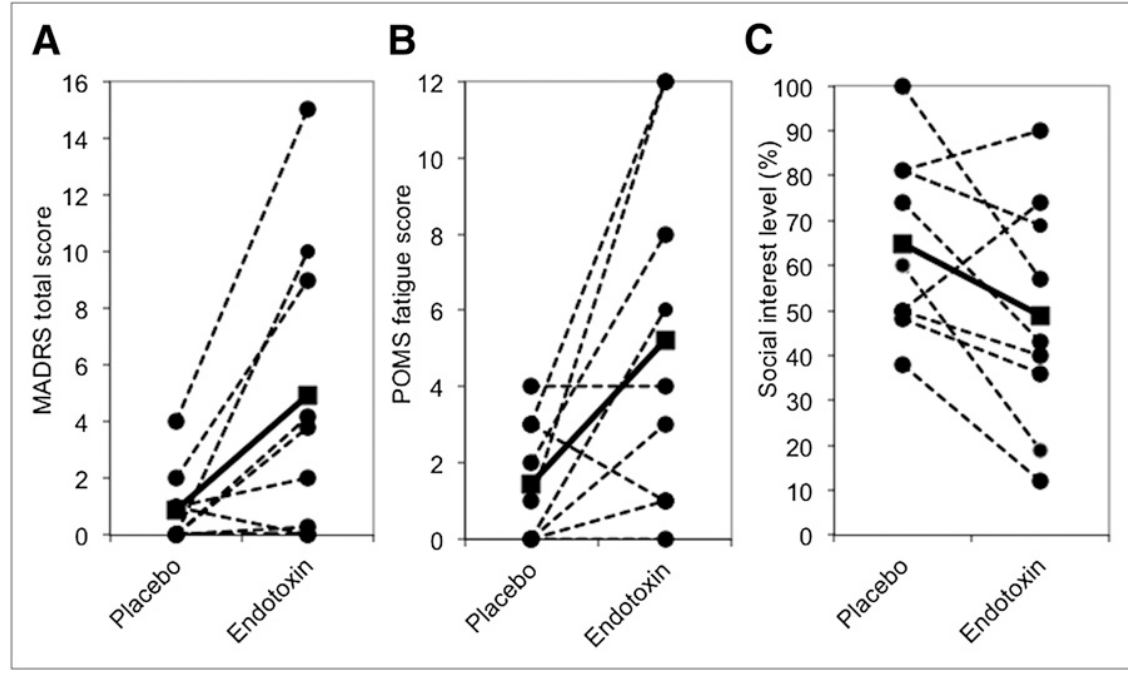

FIGURE 1. Endotoxin-induced changes in behavioral measures. (A) MADRS scores were higher in most subjects $2 \mathrm{~h}$ after endotoxin administration than in placebo. (B) Profile of Mood States Fatigue (POMS) score was higher in most subjects $3 \mathrm{~h}$ after endotoxin administration. (C) Social interest was lower in most subjects $3 \mathrm{~h}$ after endotoxin administration. Dotted lines represent individual subjects; solid line represents mean. 
social interest $\left(F_{4,72}=2.7, P=0.04\right)$, due to decreased social interest after endotoxin administration (Fig. 1C). Endotoxin administration was associated with an increase in myalgia (nonparametric, interaction: ATS, 6.5; numerator degrees of freedom, 2.6; $P=0.0005$ ) and headaches (nonparametric: ATS, 3.0; numerator degrees of freedom, $1 ; P=0.09)$. There were no order effects on behavioral measures.

\section{ROI Analysis}

The mean dose of ${ }^{18} \mathrm{~F}-\mathrm{FDG}$ administered was $189.4 \pm$ 7.4 MBq on the endotoxin day and 199.7 $\pm 7.4 \mathrm{MBq}$ on the placebo day; this difference occurred by chance and was not statistically significant ( $t$ test $P=0.77$ ). There was no significant difference in whole-brain CMRGlu between the 2 conditions (endotoxin, $5.55 \pm 0.68 \mathrm{mg} \cdot \mathrm{min} \cdot 100 \mathrm{~mL}-1$; placebo, $5.50 \pm 0.83 \mathrm{mg} \cdot \mathrm{min} \cdot 100 \mathrm{~mL}^{-1} ; t$ test $\left.P=0.88\right)$. Therefore, NGM values were used to examine regional differences and to increase statistical power. The results for the 3 a priori ROIs and for subregions of the insula and cingulate are reported in Table 1. Endotoxin administration was associated with a condition-by-region effect $\left(F_{1,40}=6.1, P=0.005\right)$ due to placebo-endotoxin differences in NGM in 2 of the a priori ROIs: In the insula (Figs. $2 \mathrm{~A}$ and $2 \mathrm{~B})$, NGM was $3.0 \% \pm 3.6 \%$ higher after endotoxin administration than with placebo $\left(F_{1,40}=6.3\right.$, corrected $P=0.048$ ), whereas in the cingulate (Fig. 2C), NGM was $2.5 \% \pm 3.2 \%$ lower after endotoxin administration $\left(F_{1,40}=5.8\right.$, corrected $\left.P=0.06\right)$. There was no significant placebo-endotoxin difference in NGM in the amygdala $\left(F_{1,40}=0.1, P=0.79\right)$.
Given the role of the right anterior insula in negative affect (27), we examined insula subregions (anterior, posterior, left, and right). Although the condition-by-regionby-side interaction did not reach significance $\left(F_{1,56}=\right.$ 2.4, $P=0.12$ ), post hoc tests showed a trend-effect of condition in the right anterior insula $\left(F_{1,56}=5.7\right.$, corrected $P=0.08$ ), because NGM was $6.1 \% \pm 10.9 \%$ higher in the right anterior insula in the endotoxin condition than in the placebo condition (Table 1; Figs. 2B and 3A).

Given the regional specializations within the cingulate, we examined cingulate subregions (anterior, middle, posterior, left, and right). Although the condition-by-regionby-side interaction was not significant $\left(F_{2,88}=1.3, P=\right.$ 0.28 ), post hoc tests showed a significant effect of condition in the right anterior cingulate $\left(F_{1,88}=14.6\right.$, corrected $P=$ 0.001 ), because NGM in the right anterior cingulate was $4.5 \% \pm 2.7 \%$ lower in the endotoxin condition than in the placebo condition (Table 1; and Figs. 2D, 3C, and 3D). There were no scan-order effects on changes in NGM in the ROIs. Exploratory SPM analysis identified several regions that might be affected by systemic inflammation (Supplemental Table 1). Some of these regions were confirmed using ROI analysis (Supplemental Table 2), including the right ventrolateral thalamus (Fig. 3A) and the left putamen (Supplemental Fig. 1).

\section{Correlational Analyses}

Correlational analyses between NGM in the insula or cingulate and behavioral or immune parameters were performed. There was a negative correlation between change in social interest and peak TNF $\alpha(r=-0.92, P=0.0005)$ and IL-6 ( $r=-0.85, P=0.004)$ - that is, higher cytokine

TABLE 1

$\mathrm{ROI}$ Analysis

\begin{tabular}{|c|c|c|c|c|c|c|c|c|}
\hline Region & Placebo NGM & Endotoxin NGM & Mean \% difference & $F_{1,40}$ & $F_{1,88}$ & $F_{1,56}$ & Uncorrected $P$ & Corrected $P$ \\
\hline \multicolumn{9}{|l|}{ Primary } \\
\hline Amygdala & $0.72 \pm 0.07$ & $0.71 \pm 0.07$ & $-0.5 \pm 11$ & 0.1 & & & 0.79 & 1 \\
\hline Cingulate & $1.24 \pm 0.07$ & $1.21 \pm 0.06$ & $-2.5 \pm 3.2$ & 5.8 & & & 0.020 & 0.060 \\
\hline Insula & $1.19 \pm 0.03$ & $1.23 \pm 0.02$ & $3.0 \pm 3.6^{\star}$ & 6.3 & & & 0.016 & $0.048^{\star}$ \\
\hline \multicolumn{9}{|l|}{ Subregions } \\
\hline \multicolumn{9}{|l|}{ Cingulate } \\
\hline Anterior right & $1.23 \pm 0.06$ & $1.17 \pm 0.07$ & $-4.5 \pm 2.7^{\star}$ & & 14.6 & & 0.0002 & $0.0012^{*}$ \\
\hline Anterior left & $1.23 \pm 0.10$ & $1.25 \pm 0.07$ & $2.0 \pm 4.4$ & & 2.2 & & 0.14 & 0.84 \\
\hline Middle right & $1.30 \pm 0.07$ & $1.26 \pm 0.08$ & $-3.4 \pm 6.3$ & & 3.4 & & 0.07 & 0.42 \\
\hline Middle left & $1.23 \pm 0.12$ & $1.20 \pm 0.08$ & $-2.3 \pm 5.3$ & & 1.7 & & 0.20 & 1 \\
\hline Posterior right & $1.25 \pm 0.13$ & $1.19 \pm 0.17$ & $-4.3 \pm 11$ & & 1.2 & & .27 & 1 \\
\hline Posterior left & $1.01 \pm 0.14$ & $0.99 \pm 0.22$ & $-2.8 \pm 18$ & & 0.3 & & 0.60 & 1 \\
\hline \multicolumn{9}{|l|}{ Insula } \\
\hline Anterior right & $1.24 \pm 0.08$ & $1.30 \pm 0.06$ & $5.4 \pm 9.1$ & & & 5.7 & 0.0204 & 0.082 \\
\hline Anterior left & $1.26 \pm 0.05$ & $1.29 \pm 0.05$ & $2.8 \pm 4.3$ & & & 1.7 & 0.198 & 0.79 \\
\hline Posterior right & $1.08 \pm 0.08$ & $1.12 \pm 0.07$ & $4.2 \pm 6.5$ & & & 2.4 & 0.124 & 0.50 \\
\hline Posterior left & $1.09 \pm 0.08$ & $1.07 \pm 0.10$ & $-1.9 \pm 6.3$ & & & 0.7 & 0.418 & 1 \\
\hline
\end{tabular}

*Differences were considered significant at corrected $P<0.05$ level.

Mean values $( \pm S D)$ for NGM for each region and both conditions are shown. Mean percentage difference was mean of each individual subject's percentage difference between placebo and endotoxin. 


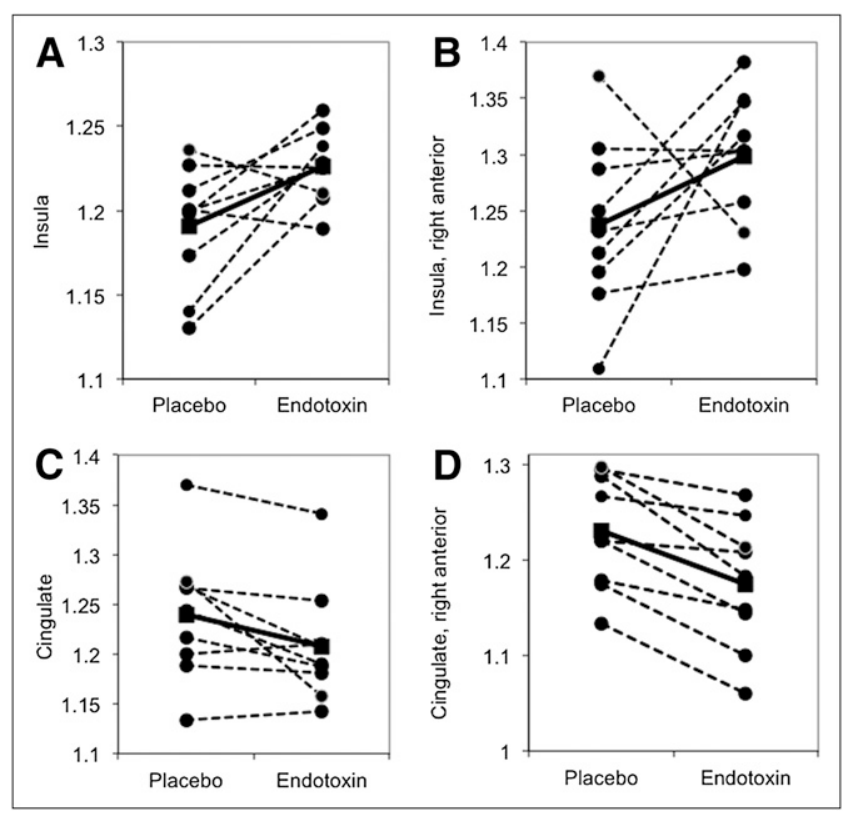

FIGURE 2. Within-subject differences in NGM in 3 primary ROls. In insula (A), NGM was higher in endotoxin condition, especially in right anterior insula (B). In cingulate (C), NGM was lower in endotoxin condition, at trend level; this effect was especially prominent in right anterior cingulate (D). Dotted lines represent individual subjects; solid line represents mean.

levels were associated with reduced social interest (Fig. 4A). There was a positive correlation between the change in social interest and change in NGM in the insula $(r=$ $0.72, P=0.029$ ) - that is, the more NGM increased, the less pronounced was the reduction in social interest produced by endotoxin (Fig. 4B). Lastly, there was a negative
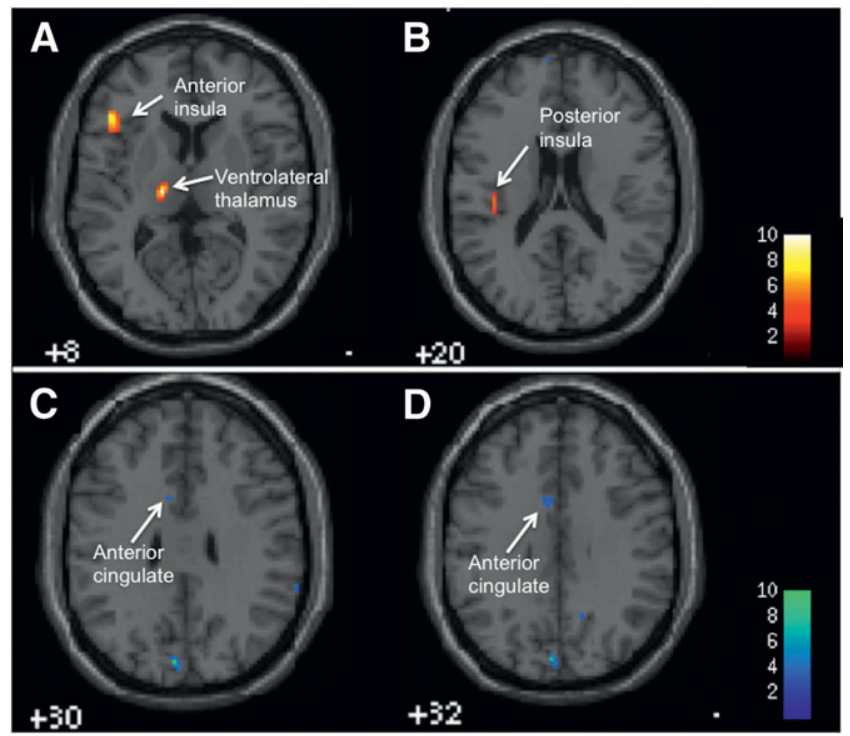

FIGURE 3. Axial multislice images from SPM showing differences in glucose metabolism in right anterior insula (A), right ventrolateral thalamus (A), right posterior insula $(B)$, and right anterior cingulate (C and D). Colors correspond to $T$ values at voxel level. Increased metabolism (endotoxin minus placebo) is displayed in yellow-red, whereas decreased metabolism (placebo minus endotoxin) is displayed in blue. correlation between peak levels of TNF $\alpha$ and IL-6 and change in NGM in the insula $(r=-0.83, P=0.005$ for TNF $\alpha$ and $r=-0.86, P=0.003$ for IL-6). That is, higher cytokine levels were associated with less endotoxin-induced increase in NGM in the insula (Fig. 4C). Despite reported associations between interferon- $\alpha$-induced fatigue and glucose metabolism in the putamen (15), we did not find any correlations between fatigue and NGM in the left putamen. There were no statistically significant correlations between cytokine levels or behavioral changes and NGM in the cingulate. There were no significant correlations between myalgia or headache and fatigue or social interest, indicating that fatigue and the reduction in social interest could not be explained by physical discomfort. There was no correlation between myalgia or headaches and cytokine levels.

\section{DISCUSSION}

We used ${ }^{18}$ F-FDG PET to measure changes in glucose metabolism in the human brain during endotoxin-induced systemic inflammation, and we found changes in NGM in the insula and, at a trend level, in the cingulate consistent with our a priori hypothesis. Secondary analyses suggest that the main effect occurred in the right anterior insula and right anterior cingulate. Exploratory SPM analysis identified other regions that may be affected by systemic inflammation. The consistency of inflammation-induced changes in NGM across subjects was striking. Seven of 9 subjects had an increase in NGM in the insula and a decrease in NGM in the cingulate, and 9 of 9 subjects had a decrease in NGM in the right anterior cingulate, suggesting that systemic inflammation induces fundamental physiologic changes in regional brain glucose metabolism.

The insula receives information about inflammatory processes in the body (27). The predominant effect seen in the right anterior insula is consistent with the fact that stimuli that drive sympathetic activity and negative affect are associated with activation in this region (27). Systemic inflammatory signals also modulate anterior cingulate functioning $(12,14)$, and positive emotionality in healthy subjects correlates with baseline metabolism in the anterior cingulate (28) — consistent with our finding that systemic inflammation (which reduces positive mood in healthy subjects) is associated with reduced metabolism in the right anterior cingulate. On the other hand, some studies have found higher neuronal activity in the subcallosal anterior cingulate in depressed subjects (29); however, whether this increase is a primary abnormality or a compensatory increase due to the decreased size of the anterior cingulate remains to be determined (30). Because the subjects examined here were euthymic with no history of depression, our results are more in line with the recent findings of Volkow et al. (28).

Contrary to our hypothesis, there was no change in NGM in the amygdala. This finding is consistent with the fact that endotoxin administration does not produce anxiety in human 
FIGURE 4. Correlations between peak cytokine levels, changes in NGM in insula, and change in social interest. (A) Peak cytokine levels were negatively correlated with change in social interest. (B) Change in social interest was positively correlated with change in NGM in insula. (C) Peak cytokine levels were negatively correlated with change in NGM in insula. Vertical axes that display cytokine levels ( $A$ and $C$ ) are logarithmic, allowing TNF $\alpha$ and IL-6 to be displayed simultaneously.

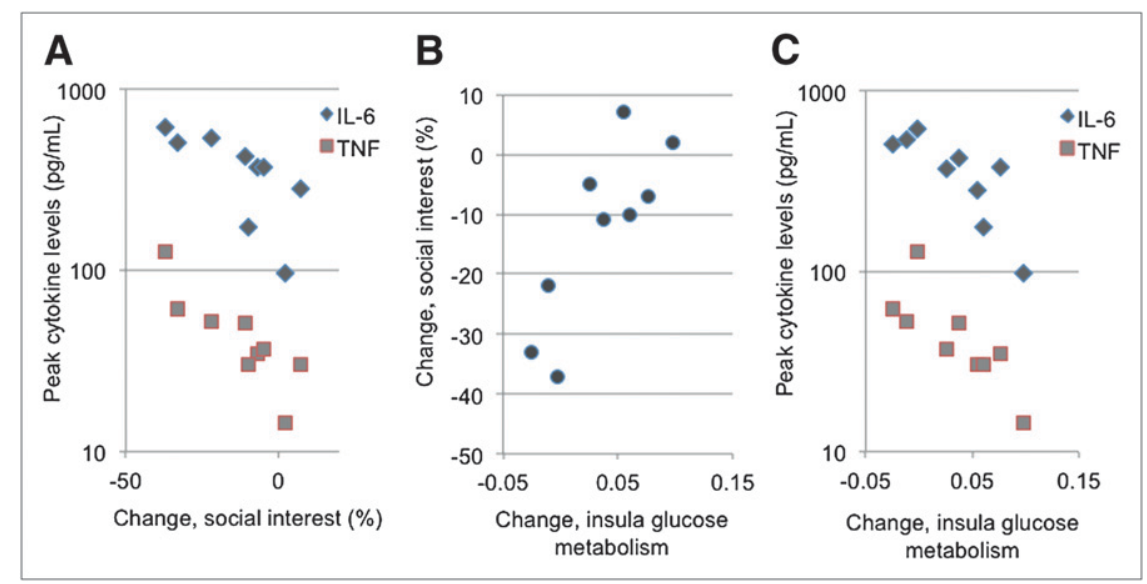

subjects (11). Overall, the brain regions susceptible to the effects of systemic inflammation identified here, including those identified with SPM (Supplemental Tables 1 and 2), are consistent with other studies on the effect of acute inflammatory stimuli on the human brain $(10,12,13)$ and with studies of patients with depression (30). Moreover, the magnitude of change in NGM (3\%-6\%) is similar to studies comparing subjects with depression with healthy controls $(31,32)$.

The immune response and the behavioral changes that occur during systemic inflammation are consistent with results from a separate cohort (11) and with data from other studies $(9,10)$. The negative correlation between peak cytokine levels and self-reported social interest is consistent with other studies (33). The positive correlation between the change in NGM in the insula and the change in social interest (Fig. 4B) and the negative correlation between the change in NGM in the insula and peak cytokine levels (Fig. 4C) were unexpected. The interoceptive information the insula receives is used to modulate peripheral responses to stress: through its projections to the hypothalamus, anterior cingulate, orbitofrontal cortex, and parabrachial nucleus (27), the insula modulates autonomic and neuroendocrine activity $(34,35)$. For instance, electric stimulation of the right insula causes increased sympathetic output (36). Because the autonomic nervous system can modulate systemic inflammatory responses $(6)$, one possible explanation for these correlations is that increased NGM in the insula is a marker for active inhibition of systemic cytokine production. That is, in subjects with higher NGM in the insula, serum cytokine levels may have been lower because of an active inhibition of the systemic inflammatory response. Lower cytokine levels may, in turn, have caused less reduction in social interest. In subjects with lower NGM in the insula, serum cytokine levels may not have been inhibited to the same extent by the brain, allowing higher TNF $\alpha$ and IL- 6 levels to cause more reduction in social interest. Because of the limited sample size, it is not possible to test this hypothesis statistically. If this is the explanation for the correlations we found, this modu- lation most likely occurred through autonomic nervous system activity and not hypothalamic-pituitary-adrenal axis activity, because there was no correlation between cytokine and cortisol levels or between cortisol levels and changes in NGM in the insula.

The negative correlation between cytokine levels and NGM in the insula conflicts with the findings of Eisenberger et al. that increases in IL-6 correlated positively with increased activity in the insula after endotoxin administration (10). This difference may be due to imaging modality ( ${ }^{18}$ F-FDG PET vs. functional MRI), to timing (90 vs. $120 \mathrm{~min}$ after endotoxin), and, most importantly, to the fact that Eisenberger et al. found a difference in activity in the insula only when subjects participated in a task of social exclusion, whereas in our study subjects were at rest in the PET camera. That is, the difference in NGM in the insula in our subjects can be attributed only to endotoxin administration.

There are several limitations to this study. The small sample size may have led to a type II error. For instance, the lack of a statistically significant effect of endotoxin on heart rate and temperature is likely a type II error, because these are known effects of endotoxin administration (18).

Although the MADRS score increased after endotoxin administration, this was mostly due to the lassitude item, and we did not find a significant effect on the MADRS mood item. Others have found a reduction in mood after endotoxin administration, but this effect was reduced when controlling for fatigue (33), which is consistent with our data. It is possible that negative affect after endotoxin administration is due to physical discomfort; however, there was no correlation between physical symptoms and depressive symptoms.

Because endotoxin has subjective effects, the masking of the study may have been compromised, possibly affecting behavioral ratings, but it is unlikely that this would have an effect on brain glucose metabolism.

Although subjects were asked to not take any nonsteroidal antiinflammatory medications for $48 \mathrm{~h}$ before each imaging session, it is possible that subjects took such medi- 
cations without reporting it. It is also possible that subjects took naproxen, which, unlike ibuprofen and acetaminophen, has a long half-life and could have an antiinflammatory effect.

Lastly, in this study $80 \%$ of subjects were men, possibly limiting the generalizability of these results to women.

\section{CONCLUSION}

In this within-subject study, we showed that endotoxin administration in humans at rest is associated with changes in NGM in 2 brain regions implicated in the pathophysiology of depression: insula and cingulate. These regions are also involved in interoception, positive emotionality, and motivation. Correlational analyses suggest that the insula may participate in the modulation of systemic levels of inflammatory cytokines, which is consistent with the known functions of the insula; this is a finding with important potential implications for depression that we plan to address in future studies.

\section{DISCLOSURE STATEMENT}

The costs of publication of this article were defrayed in part by the payment of page charges. Therefore, and solely to indicate this fact, this article is hereby marked "advertisement" in accordance with 18 USC section 1734.

\section{ACKNOWLEDGMENTS}

We thank the Yale PET Center staff, the pharmacy, and the subjects who participated in this study. Research was made possible by the Connecticut Department of Mental Health and Addiction Services. A portion of this study was supported by grants K12DA00167 and UL1 RR024139 (Clinical Translational Science Awards from the National Center for Research Resources, a component of the National Institutes of Health, and National Institutes of Health roadmap for Medical Research). No other potential conflict of interest relevant to this article was reported.

\section{REFERENCES}

1. Wager-Smith K, Markou A. Depression: a repair response to stress-induced neuronal microdamage that can grade into a chronic neuroinflammatory condition? Neurosci Biobehav Rev. 2011;35:742-764.

2. Krishnan V, Nestler EJ. The molecular neurobiology of depression. Nature. 2008;455:894-902.

3. Shelton RC, Claiborne J, Sidoryk-Wegrzynowicz M, et al. Altered expression of genes involved in inflammation and apoptosis in frontal cortex in major depression. Mol Psychiatry. 2011;16:751-762.

4. Dowlati Y, Herrmann N, Swardfager W, et al. A meta-analysis of cytokines in major depression. Biol Psychiatry. 2010;67:446-457.

5. Capuron L, Fornwalt FB, Knight BT, Harvey PD, Ninan PT, Miller AH. Does cytokine-induced depression differ from idiopathic major depression in medically healthy individuals? J Affect Disord. 2009;119:181-185.

6. DellaGioia N, Hannestad J. A critical review of human endotoxin administration as an experimental paradigm of depression. Neurosci Biobehav Rev. 2010;34: $130-143$.

7. De La Garza R, 2nd. Endotoxin- or pro-inflammatory cytokine-induced sickness behavior as an animal model of depression: focus on anhedonia. Neurosci Biobehav Rev. 2005;29:761-770.
8. Bucks RS, Gidron Y, Harris P, Teeling J, Wesnes KA, Perry VH. Selective effects of upper respiratory tract infection on cognition, mood and emotion processing: a prospective study. Brain Behav Immun. 2008;22:399-407.

9. Reichenberg A, Yirmiya R, Schuld A, et al. Cytokine-associated emotional and cognitive disturbances in humans. Arch Gen Psychiatry. 2001;58:445-452.

10. Eisenberger NI, Inagaki TK, Rameson LT, Mashal NM, Irwin MR. An fMRI study of cytokine-induced depressed mood and social pain: the role of sex differences. Neuroimage. 2009;47:881-890.

11. Hannestad J, DellaGioia N, Ortiz N, Pittman B, Bhagwagar Z. Citalopram reduces endotoxin-induced fatigue. Brain Behav Immun. 2011;25:256-259.

12. Rosenkranz MA, Busse WW, Johnstone T, et al. Neural circuitry underlying the interaction between emotion and asthma symptom exacerbation. Proc Natl Acad Sci USA. 2005;102:13319-13324.

13. Harrison NA, Brydon L, Walker C, et al. Neural origins of human sickness in interoceptive responses to inflammation. Biol Psychiatry. 2009;66:415-422.

14. Harrison NA, Brydon L, Walker C, Gray MA, Steptoe A, Critchley HD. Inflammation causes mood changes through alterations in subgenual cingulate activity and mesolimbic connectivity. Biol Psychiatry. 2009;66:407-414.

15. Capuron L, Pagnoni G, Demetrashvili MF, et al. Basal ganglia hypermetabolism and symptoms of fatigue during interferon-alpha therapy. Neuropsychopharmacology. 2007;32:2384-2392.

16. Brydon L, Harrison NA, Walker C, Steptoe A, Critchley HD. Peripheral inflammation is associated with altered substantia nigra activity and psychomotor slowing in humans. Biol Psychiatry. 2008;63:1022-1029.

17. Eisenberger NI, Berkman ET, Inagaki TK, Rameson LT, Mashal NM, Irwin MR. Inflammation-induced anhedonia: endotoxin reduces ventral striatum responses to reward. Biol Psychiatry. 2010;68:748-754.

18. Lowry SF. Human endotoxemia: a model for mechanistic insight and therapeutic targeting. Shock. 2005;24(suppl 1):94-100.

19. American Psychiatric Association. Diagnostic and Statistical Manual of Mental Disorders: DSM-IV-TR. 4th ed. Washington, DC: American Psychiatric Association; 2000 .

20. Moore DF, Altarescu G, Barker WC, Patronas NJ, Herscovitch P, Schiffmann R. White matter lesions in Fabry disease occur in 'prior' selectively hypometabolic and hyperperfused brain regions. Brain Res Bull. 2003;62:231-240.

21. Tzourio-Mazoyer N, Landeau B, Papathanassiou D, et al. Automated anatomical labeling of activations in SPM using a macroscopic anatomical parcellation of the MNI MRI single-subject brain. Neuroimage. 2002;15:273-289.

22. Holmes CJ, Hoge R, Collins L, Woods R, Toga AW, Evans AC. Enhancement of MR images using registration for signal averaging. J Comput Assist Tomogr. 1998;22:324-333.

23. Makris N, Goldstein JM, Kennedy D, et al. Decreased volume of left and total anterior insular lobule in schizophrenia. Schizophr Res. 2006;83:155-171.

24. Wells WM, 3rd, Viola P, Atsumi H, Nakajima S, Kikinis R. Multi-modal volume registration by maximization of mutual information. Med Image Anal. 1996;1:35-51.

25. Norcross JC, Guadagnoli E, Prochaska JO. Factor structure of the Profile of Mood States (POMS): two partial replications. J Clin Psychol. 1984;40:1270-1277.

26. Bandelow B, Brunner E, Beinroth D, et al. Application of a new statistical approach to evaluate a clinical trial with panic disorder patients. Eur Arch Psychiatry Clin Neurosci. 1999;249:21-27.

27. Craig AD. Emotional moments across time: a possible neural basis for time perception in the anterior insula. Philos Trans R Soc Lond B Biol Sci. 2009;364: 1933-1942.

28. Volkow ND, Tomasi D, Wang GJ, et al. Positive emotionality is associated with baseline metabolism in orbitofrontal cortex and in regions of the default network. Mol Psychiatry. 2011;16:818-825.

29. Hamani C, Mayberg H, Stone S, Laxton A, Haber S, Lozano AM. The subcallosal cingulate gyrus in the context of major depression. Biol Psychiatry. 2011; 69:301-308.

30. Price JL, Drevets WC. Neurocircuitry of mood disorders. Neuropsychopharmacology. 2010;35:192-216.

31. Drevets WC, Price JL, Simpson JR Jr, et al. Subgenual prefrontal cortex abnormalities in mood disorders. Nature. 1997;386:824-827.

32. Biver F, Goldman S, Delvenne V, et al. Frontal and parietal metabolic disturbances in unipolar depression. Biol Psychiatry. 1994;36:381-388.

33. Eisenberger NI, Inagaki TK, Mashal NM, Irwin MR. Inflammation and social experience: an inflammatory challenge induces feelings of social disconnection in addition to depressed mood. Brain Behav Immun. 2010;24:558-563.

34. Ongür D, Price JL. The organization of networks within the orbital and medial prefrontal cortex of rats, monkeys and humans. Cereb Cortex. 2000;10:206-219.

35. Jones CL, Ward J, Critchley HD. The neuropsychological impact of insular cortex lesions. J Neurol Neurosurg Psychiatry. 2010;81:611-618.

36. Oppenheimer SM, Gelb A, Girvin JP, Hachinski VC. Cardiovascular effects of human insular cortex stimulation. Neurology. 1992;42:1727-1732. 